\title{
BMJ open Emergency and surgery services of primary hospitals in the United Republic of Tanzania
}

\author{
Tom Penoyar, ${ }^{1}$ Hillary Cohen, ${ }^{2} \mathrm{P}$ Kibatala, ${ }^{3}$ A Magoda, ${ }^{4}$ G Saguti, ${ }^{3}$ L Noel, ${ }^{5}$ \\ S Groth, ${ }^{5}$ D H Mwakyusa, ${ }^{6}$ M Cherian ${ }^{5}$
}

To cite: Penoyar T, Cohen $\mathrm{H}$, Kibatala $\mathrm{P}$, et al. Emergency and surgery services of primary hospitals in the United Republic of Tanzania. BMJ Open 2012;2:e000369. doi:10.1136/

bmjopen-2011-000369

- Prepublication history for this paper is available online. To view these files please visit the journal online (http:// bmjopen.bmj.com).

Received 6 October 2011 Accepted 14 December 2011

This final article is available for use under the terms of the Creative Commons Attribution Non-Commercial 2.0 Licence; see http://bmjopen.bmj.com

For numbered affiliations see end of article.

Correspondence to DrTom Penoyar; tpenoyar@ bidmc.harvard.edu

\section{ABSTRACT}

Objective: The primary objective was to evaluate the capacity of first-referral health facilities in Tanzania to perform basic surgical procedures. The intent was to assist in planning strategies for universal access to life-saving and disability-preventing surgical services.

Design: Cross-sectional survey.

Setting: First-referral health facilities in the United Republic of Tanzania.

Participants: 48 health facilities.

Measures: The WHO Tool for Situational Analysis to Assess Emergency and Essential Surgical Care was employed to capture a health facility's capacity to perform basic surgical (including obstetrics and trauma) and anaesthesia interventions by investigating four categories of data: infrastructure, human resources, interventions available and equipment. The tool queried the availability of eight types of care providers, 35 surgical interventions and 67 items of equipment.

Results: The 48 facilities surveyed served 18.6 million residents ( $46 \%$ of the population). Supplies for basic airway management were inconsistently available. Only $42 \%$ had consistent access to oxygen, and only six functioning pulse oximeters were located in all facilities surveyed. $37.5 \%$ of facilities reported both consistent running water and electricity. While very basic interventions (suturing, wound debridement, incision and drainage) were provided in nearly all facilities, more advanced life-saving procedures including chest tube thoracostomy (30/48), open fracture management (29/48) and caesarean section delivery (32/48) were not consistently available.

Conclusions: Based on the results in this WHO country survey, significant gaps exist in the capacity for emergency and essential surgical services in Tanzania including deficits in human resources, essential equipment and infrastructure. The information in this survey will provide a foundation for evidence-based decisions in country-level policy regarding the allocation of resources and provision of emergency and essential surgical services.

\section{INTRODUCTION}

Surgical services at the first-referral level are an essential component of comprehensive primary healthcare. Conditions that can be

\section{ARTICLE SUMMARY}

Article focus
- On-site visits to primary health centres in
a developing nation.
- Evaluate capacity to deliver emergency and
surgical care-identify gaps in equipment, skills
and personnel.
Key messages
- Basic surgical procedures are being performed in
nearly all health centres.
- Significant deficits in human resources, essential
equipment and infrastructure.
- Pulse oximetry is rarely available.
Strengths and limitations of this study
- Most comprehensive evaluation of a developing
country's surgical capacity.
- Based on established well-accepted analysis tool.
- Relies on subjective measures and estimate.

treated with surgery account for an estimated $11 \%$ of the world's disability-adjusted life years. ${ }^{1}$ Despite recent data estimating the global volume of surgery at 234 million surgical procedures annually and significant disparities between procedures performed in high- and low-income counties, global public health initiatives have traditionally neglected the necessity for the provision of surgical services. $^{2}$ Poor access to surgical services, particularly at rural facilities, results in excess morbidity and mortality from a broad range of treatable surgical conditions including injuries, complications of pregnancy, sequelae of infectious diseases, acute abdominal conditions and congenital anomalies. Improving the access to surgical services in low-income countries requires a systems-based approach addressing gaps in infrastructure, trained/skilled personnel, appropriate equipment and medications.

Tanzania, similar to other sub-Saharan African countries, faces significant challenges in the provision of health services. Infant mortality is 68 per 1000 live births and 
maternal mortality rate is 578 per 100000 live births. ${ }^{3}$ The leading causes of maternal death (haemorrhage, unsafe abortion, eclampsia and obstructed labour) can all be addressed with appropriate emergency obstetric care, which often require surgical and/or anaesthesia interventions. In a 1999 Tanzanian Ministry of Health and Social Welfare (MoHSW) census, health facilities numbered 4714 with 280 hospitals, 479 health centres and 3955 dispensaries for a total of 32000 beds (1:896 people). There were 110 surgeons $(1 / 3$ in cities, $1 / 3$ in administration and 1/3 emigrated) and 16 anaesthesiologists. Human resources for health were critically absent, with fewer than $1 / 3$ of posts filled in primary hospitals. ${ }^{4}$

As funders and public health experts adopt the expansion of primary healthcare services, the inclusion of surgical services at the first-referral level is critical. The purpose of this survey was to collect knowledge gained from comprehensive quantitative assessments of surgical capacity in sub-Saharan African countries such as Tanzania in order to assist in planning strategies for universal access to life-saving and disability-preventing surgical services.

\section{MATERIALS AND METHODS}

The WHO Tool for Situational Analysis to Assess Emergency and Essential Surgical Care was developed as a comprehensive questionnaire to quantify the surgical capacity in a wide range of health facilities. ${ }^{5}$ This online tool captures a health facility's capacity to perform basic surgical (including obstetrics and trauma) and anaesthesia interventions by investigating four categories of data: infrastructure, human resources, interventions available and equipment. The tool queries the availability of eight types of care providers, 35 surgical interventions and 67 items of equipment.

WHO situation analysis tool to assess Emergency and Essential Surgical Care was completed at 48 health facilities representing 16 of 26 regions in Tanzania. The health facility data were obtained during site visits by representatives from the Tanzania MoHSW, WHO country office and members of Global Initiative for Emergency and Essential Surgical Care (GIEESC) between March 2009 and October 2010. Data on various indices were entered into and analysed from WHO Global DataCol Database for Emergency and Essential Surgical Care (table 1). Some results, such as the average distance travelled prior to admission, were expressed as a weighted mean to better reflect the distance travelled by the average patient seeking surgical care in the country. To calculate the weighted mean, we summed the products of annual admissions and average distance travelled for each facility and then divided by the sum of annual admissions for all facilities.

By local convention, a physician who has trained in general surgery is considered a surgical specialist. Further specialisation, such as urologic, orthopaedic or cardiothoracic surgery, is termed as super specialty. Facilities were asked the size of the 'population served', intending to quantify the population living in the catchment area. This value thus represents the number of residents who would use the facility as their firstreferral health facility, not the number of patients seen.

\section{RESULTS}

Forty-eight facilities, representing 16 of 26 regions and serving 18.6 million residents (46\% of the population), completed the WHO Integrated Management for Emergency and Essential Surgical Care (IMEESC) Situational Analysis research tool. The average population served per facility was 425000 , though five facilities served 10000 or fewer residents. A total of 9085 hospital beds were reported, averaging 189 beds per facility (range 15-350 beds). One hundred eighteen operating rooms were identified.

The weighted mean of distance travelled prior to admission was $119 \mathrm{~km}$ (74 miles). Figure 1 displays the locations of facilities with markers sized to the population served. This map demonstrates that the six facilities serving the largest population are located on the southern and northern periphery. The central regions are dominated by health facilities in rural areas serving small populations.

Annual admissions averaged 2001 per facility (range $350-5000)$. On average, $34 \%$ of all admissions required either minor or major surgical interventions.

A total of 4965 healthcare providers were reported in the 48 facilities. Sixty-four surgical specialists (ie, physicians with dedicated surgical training) were identified, and $56(88 \%)$ of identified surgical specialists were employed by the six largest hospitals. The great majority of anaesthesia providers $(176 / 203=87 \%)$ were nonphysicians, and only 11 formally trained anaesthesiologists were identified. Other medical staff providing surgical and anaesthesia services in the facilities included 4017 assistant medical officers (non-physician medical officers, paramedics and midwives).

Of the 35 basic interventions listed in the tool, only suturing was available at all facilities. Additionally, incision and drainage, male circumcision and wound debridement were widely available and provided at $98 \%$, $98 \%$ and $92 \%$ of facilities, respectively. Caesarean section was available at $67 \%$ of facilities.

Equipment was largely inadequate, including a significant gap in availability of functioning anaesthesia machines. Running water and electricity were widely available with only two facilities having no access to either water or electricity. However, only $37.5 \%$ of facilities reported both consistent running water and electricity. Greater than half of facilities reported never using eye protection and $46 \%$ reported no access to this critical piece of personal protective equipment. Six facilities had all essential equipment consistently available: Bombo Regional Hospital, Dodoma Regional Hospital, St Francis District Hospital, Ilembula Hospital, Besha Health Centre and Muhimbili National Hospital. Oxygen supplies were inconsistent in many facilities. 
Table 1 Results of Situational Analysis Tool

C I N

General and congenital

Personnel

General physician performing surgery

Non-physicians performing surgery

Paramedics and midwives

Physicians trained in surgery (specialist)

Procedure

Appendectomy

Biopsy

Burn care

Cataract repair

Cleft lip repair

Congenital hernia repair

Cystotomy

Hernia repair

Hydrocele

Incision and drainage

Laparotomy

Male circumcision

Neonatal surgery

Suturing

Tubal ligation/vasectomy

Urethral stricture

Blood bank

Electricity

113 Emergency guidelines

122 Emergency room

4017 Generator

64 Haemoglobin and urine analysis

Medical records

P Running water

69 Surgery guidelines

81 Cotton wool

90 Adhesive tape

35

25

71

63

69

88

98

75

98

35

100

71

46

Apron, plastic, reusable

Bandages sterile

Batteries for flashlight

Bucket, plastic

Capped bottle, alcohol solution

Disposable needles \# 25, 21, 19

Drum for sterile dressings

Examination table

Eye protection

Face masks

Forceps, Kocher

Forceps, artery

Gloves (non-sterile)

Gloves (sterile)

Kidney dishes, stainless steel

Light source (lamp and flashlight)

Nail brush, scrubbing

Nasogastric tubes 10 to $16 \mathrm{FG}$

Needle holder

Needles, cutting and round

Retractors

Scalpel handle with blade

Scissors blunt $14 \mathrm{~cm}$

Scissors straight $12 \mathrm{~cm}$

Sharps disposal container

Sheeting, plastic for exam table

Soap

Sterile gauze dressing

Steriliser

Suction pump (manual or electric)

Suture, synthetic absorbable

Syringes $10 \mathrm{ml}$

Syringes $2 \mathrm{ml}$

Thermometer

Towel cloth

Urinary catheter disposable \#12, 14, 18

Wash basin

Waste disposal container

$\begin{array}{lll}29 & 48 & 23\end{array}$

$\begin{array}{lll}44 & 52 & 4\end{array}$

$\begin{array}{lll}25 & 13 & 63\end{array}$

$\begin{array}{lll}33 & 15 & 52\end{array}$

$\begin{array}{lll}58 & 2 & 40\end{array}$

$\begin{array}{lll}96 & 4 & 0\end{array}$

$\begin{array}{lll}98 & 2 & 0\end{array}$

$\begin{array}{lll}56 & 35 & 8\end{array}$

$\begin{array}{lll}58 & 6 & 35\end{array}$

$\begin{array}{lll}77 & 21 & 2\end{array}$

$\begin{array}{lll}96 & 4 & 0\end{array}$

$\begin{array}{lll}81 & 15 & 4\end{array}$

$\begin{array}{lll}98 & 2 & 0\end{array}$

$\begin{array}{lll}58 & 33 & 8\end{array}$

$\begin{array}{lll}94 & 6 & 0\end{array}$

$\begin{array}{lll}79 & 13 & 8\end{array}$

$\begin{array}{lll}98 & 2 & 0\end{array}$

8388

$90 \quad 10 \quad 0$

$\begin{array}{lll}40 & 15 & 46\end{array}$

$\begin{array}{lll}69 & 25 & 6\end{array}$

$\begin{array}{lll}73 & 19 & 8\end{array}$

$\begin{array}{lll}81 & 10 & 8\end{array}$

9280

$\begin{array}{lll}90 & 10 & 0\end{array}$

$\begin{array}{lll}88 & 13 & 0\end{array}$

$\begin{array}{lll}73 & 17 & 10\end{array}$

$\begin{array}{lll}85 & 10 & 5\end{array}$

$\begin{array}{lll}71 & 17 & 13\end{array}$

$\begin{array}{lll}90 & 10 & 0\end{array}$

9460

$\begin{array}{lll}77 & 17 & 6\end{array}$

$94 \quad 4 \quad 2$

$83 \quad 15 \quad 2$

$\begin{array}{lll}77 & 21 & 2\end{array}$

$\begin{array}{lll}98 & 2 & 0\end{array}$

$\begin{array}{lll}65 & 23 & 13\end{array}$

$\begin{array}{lll}98 & 2 & 0\end{array}$

$\begin{array}{lll}96 & 4 & 0\end{array}$

$\begin{array}{lll}85 & 13 & 2\end{array}$

9640

$\begin{array}{lll}90 & 10 & 0\end{array}$

$\begin{array}{lll}100 & 0 & 0\end{array}$

$\begin{array}{lll}100 & 0 & 0\end{array}$

$\begin{array}{lll}96 & 4 & 0\end{array}$

$\begin{array}{lll}85 & 13 & 2\end{array}$

$\begin{array}{lll}58 & 33 & 8\end{array}$

$94 \quad 4 \quad 2$

9820

Anaesthesia guidelines

$\begin{array}{lll}27 & 4 & 69\end{array}$

Anaesthesia machine

Personnel

General practitioners performing

Blood pressure measuring equipment

$\begin{array}{lll}67 & 0 & 33\end{array}$

9820

aesthesia

176 Cricothyroidotomy set

$\begin{array}{lll}27 & 21 & 52\end{array}$

Non-physicians performing anaesthesia

Cricothyroidotomy set
Endotracheal tubes, cuffed sizes 5.5 to 9

$\begin{array}{lll}65 & 8 & 27\end{array}$

Endotracheal tubes, uncuffed sizes 3.0 to 5.0 


\section{Table 1 Continued}

Procedure

Airway foreign body

Cricothyroidotomy

General anaesthesia

Ketamine IV

Regional anaesthesia

Resuscitation

Spinal anaesthesia

\section{Orthopaedics and traumatology}

Procedure

Chest tube placement

Clubfoot repair

Contracture release

Debridement

Fracture management, closed

Fracture management, open

Joint dislocation reduction

Limb amputation

Osteomyelitis/septic arthritis

Obstetrics/gynaecology

Personnel

Physicians trained in OBGYN (specialists) $\quad 74$

Procedure

Caesarean delivery

Dilation and curettage

Obstetric fistula repair

\section{$\mathrm{P}$}

83

44

65

67

42

88

77
IV cannula sizes $18,22,24$

IV infusion set

IV Infusor bags

Laryngoscope handle

Laryngoscope Macintosh blades (adult)

Laryngoscope Macintosh blades (paediatric)

Magills forceps (adult)

Magills forceps (paediatric)

Mask and tubing to connect to oxygen supply

Oropharyngeal airway (adult)

Oropharyngeal airway (paediatric)

Oxygen concentrator

Oxygen cylinder

Pain management guidelines

Post-operative recovery room

Pulse oximetry

Resuscitator bag valve and mask (adult)

Resuscitator bag valve and mask (paediatric)

Scalp vein infusion set

Spare bulbs and batteries for laryngoscope

Stethoscope

Suction catheter sizes $16 \mathrm{Fr}$

Tongue depressor, wooden, disposable

\section{Radiography}

\section{$P$}

63

35

33

92

88

61

92

65

63

\section{Vaginal speculum}

Twenty facilities (42\%) had uninterrupted access to oxygen, with most relying on oxygen concentrators. Fifteen facilities (32\%) had no access to an anaesthesia machine of any kind. Of all facilities surveyed, only six pulse oximeters were located. In Tanzania, the regional blood bank system is independent of any hospital facility and $77 \%$ of facilities reported having a blood bank. The x-ray was fully functional in $33 \%$ of facilities and interrupted in $44 \%$, leaving $23 \%$ of facilities with no radiographic capacity. All facilities have access to haemoglobin and urine analysis testing.
Complete results from the evaluation are shown in table 1. Information was placed into one of four mutually exclusive and comprehensive medical fields. For simplification, in table 1, laboratory tests and other infrastructure (ie, blood bank, electricity) were included under equipment.

\section{DISCUSSION}

More than 5 million people die from injuries every year and many more are left with permanent disabilities. Significant disparities in care exist between high- and 
Figure 1 Facilities evaluated. Ring size proportional to population served.

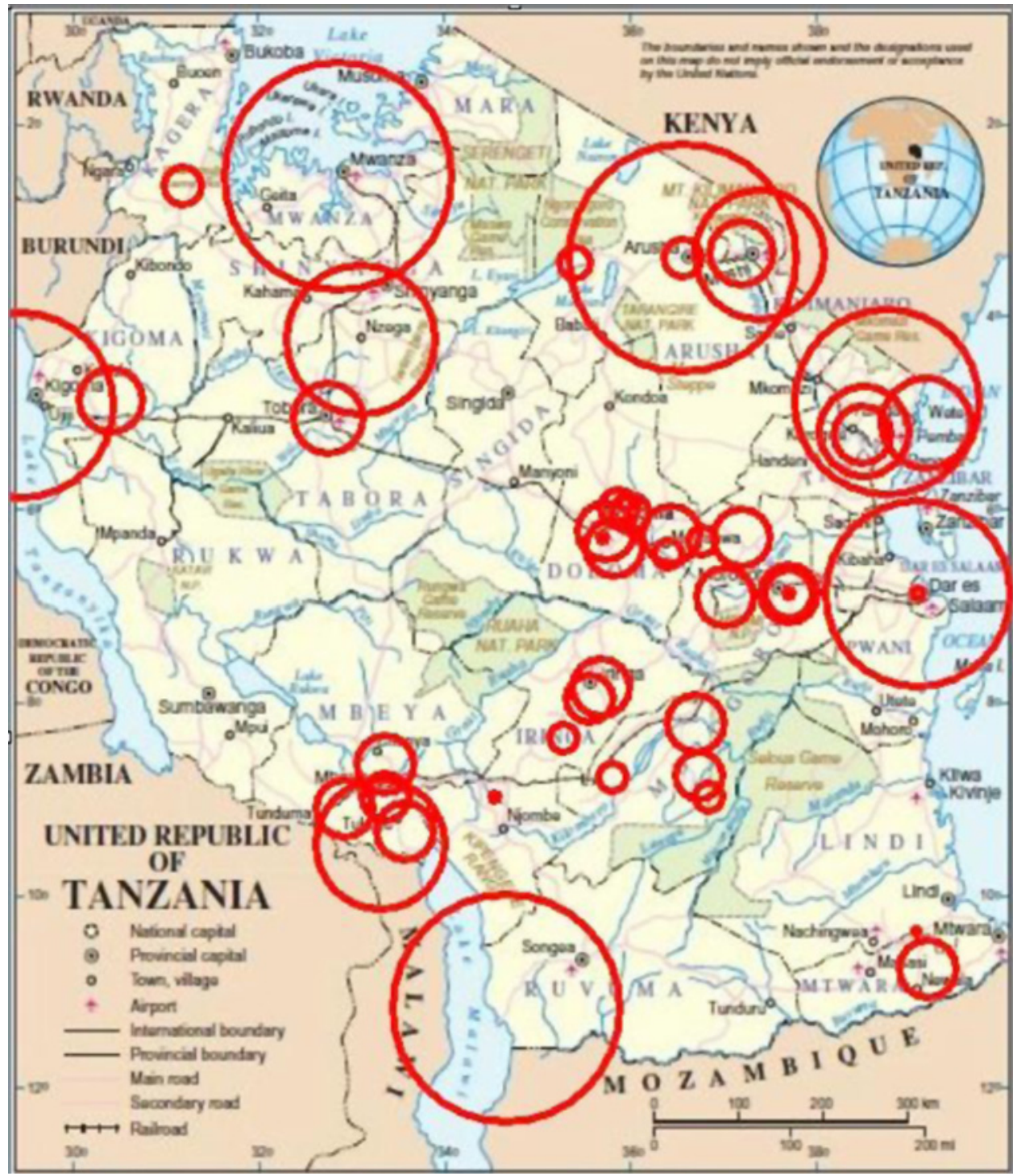

low-income countries for patients with surgically treatable conditions. An estimate of the global burden of surgery showed that only $26 \%$ of estimated surgical procedures were performed in low-income countries, despite these countries accounting for $70 \%$ of the global population. ${ }^{2}$ Of the estimated 536000 maternal deaths in 2005, developing countries accounted for $99 \%$ of these deaths ${ }^{6}$; much of this mortality could be prevented by timely access to emergency and basic surgical services.

The provision of surgical services has historically been neglected in public health programmes. ${ }^{7}$ It is often assumed that surgery and anaesthesia interventions are expensive, technologically demanding and can only be delivered in large hospitals and by specialists. However, limiting surgical care to large facilities in developing countries makes it inaccessible to the large segment of the population in decentralised areas. Experience shows that basic surgical services can be cost-effective and safely delivered even in settings with limited resources. ${ }^{8}$

Two studies have examined the cost-effectiveness of small hospitals performing basic surgical operations in resource poor settings. ${ }^{9} 10$ The cost per DALY averted in each study for all patients seen was US $\$ 10.93$ and US $\$ 32.78$. Although these studies did not separate surgical from non-surgical patients in calculating cost/DALY, both hospitals had a significant percentage $(29 \%-67 \%)$ of surgical diagnoses contributing to the calculation. These costs compare favourably with other primary health interventions in developing countries. ${ }^{1}$

WHO developed the IMEESC toolkit that has been implemented in 37 countries including Tanzania in January 2007.5 Targeted activities to improve surgical capacity have included the formation of a formal 'Surgical Task force' in Tanzania MoHSW, training courses, the adoption of IMEESC toolkit by the Tanzania Surgical Association and hosting the biennial WHO GIEESC meeting in Dar es Salaam, Tanzania.

WHO GIEESC was established in 2005 as a collaboration of local and international organisations, academia, health authorities and WHO, in response to the recognition of surgery as a critical component of population based health. ${ }^{5}$ The research arm of WHO GIEESC developed WHO situational analysis tool to provide data in surgical care capacity to assist ministries of health in 
low- and middle-income countries for making evidencebased improvements.

This study provides an overview of the capacity for surgical care in 16 regions of Tanzania and demonstrates the significant gaps in infrastructure, human resources, life-saving and disability-preventive surgical interventions and essential equipment.

Despite the introduction of WHO programme for emergency and essential surgical care in Tanzania in 2007 and the efforts by the Tanzanian MoHSW to train non-physicians to deliver select surgical services such as caesarean sections, skilled health personnel to deliver surgical services remain inadequate for a significant portion of the country. This deficit is most pronounced in the rural areas, where patients travel great distances to reach health facilities and consequently face significant delays in care.

Although most facilities had a functioning operating theatre, fewer than half had uninterrupted access to oxygen and a third of facilities did not have access to an anaesthesia machine as is seen in many sub-Saharan African countries. ${ }^{11}$ Significant improvements in surgical mortality in developed countries have resulted from improvements in the delivery of safe anaesthesia. The existing gap of safe anaesthesia services likely limits the availability of life-saving surgeries in Tanzania or results in significant complications and unnecessary patient suffering when anaesthesia is not available.

Of the 35 basic surgical interventions, many hospitals did not have the capacity to deliver all the basic services. As demonstrated in figure 2, this survey showed that facilities in the central and southern region had less capacity to provide basic surgical services. Additionally, the consistent lack of oxygen tubing, pulse oximeters and paediatric airway equipment is a significant barrier to the provision of life-saving services in the regions studied.

Delivery of surgical services is dependent on the availability of all components inherent in a functioning health system. Systematic changes that address human resources, supplies/equipment and infrastructure are necessary to improve mortality from surgically treatable conditions. The benefits of these changes will significantly impact the mortality of patients with obstetricrelated emergencies and traumatic injuries, particularly women and children. However, the efforts made to improve disease-specific surgical interventions will not

Figure 2 Rings sized on ratio of (population served: annual procedures). Large rings are underserved.

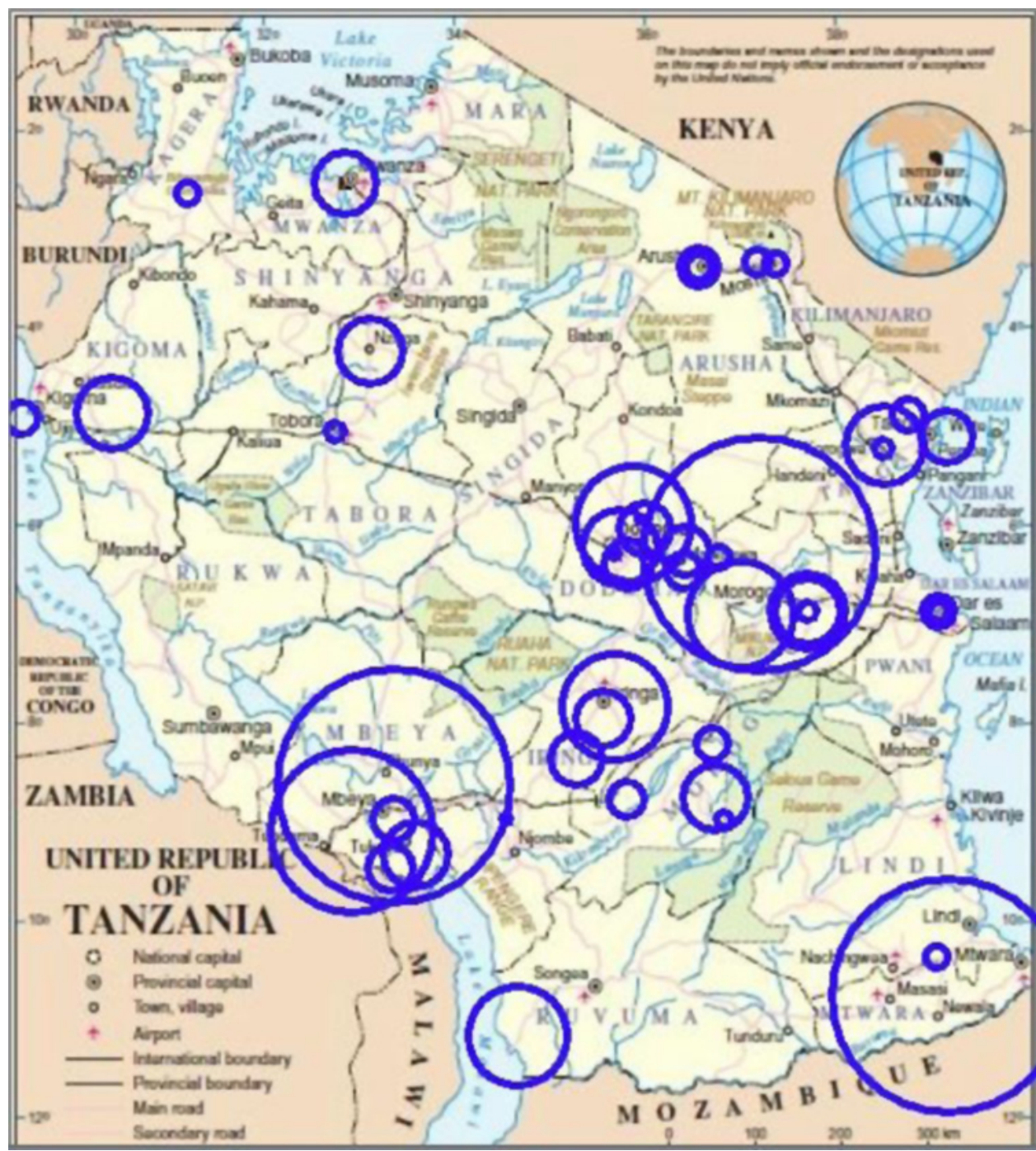


have an isolated impact on surgically treatable conditions and meet the Millennium Development Goals 4, 5 and 6. Systematic changes such as investments in oxygen and related equipment and appropriately trained surgical workforce will also serve to benefit patients suffering from a range of conditions including sepsis, pneumonia, HIV-related conditions and other infectious diseases.

There are several limitations to this survey. First, it provides only a brief overview of the capacity for surgical care and cannot be used for detailed programme planning. Second, an independent observer did not verify the answers provided in the survey by the health provider or director of the health facilities. Third, it does not capture data from every first-referral health facilities of the country.

This survey presents the first snapshot of life-saving surgical services in Tanzania using WHO Tool for Situational Analysis to Assess Emergency and Essential Surgical Care. This snapshot view provides additional evidence that investments in human resources, essential equipment and infrastructure are needed to strengthen district surgical services in Tanzania to benefit rural population. Addressing the unmet need of surgical (including anaesthesia, obstetrics and trauma) services within existing related national programmes for maternal and child health will strengthen health systems, particularly at the district level. ${ }^{12}$ These investments will have the secondary effect of improving the overall healthcare system and the treatment of many nonsurgical conditions. Further research is needed to quantify the true burden of surgical disease in Tanzania and the cost-benefit of specific interventions to improve surgical services.

\footnotetext{
Author affiliations

${ }^{1}$ Beth Israel Deaconess Medical Center, Boston, Massachusetts, USA

${ }^{2}$ Maimonides Medical Center, Brooklyn, New York, USA

${ }^{3}$ WHO Country Office, Dar es Salaam, United Republic of Tanzania

${ }^{4}$ St Francis Designated District Hospital, Ifakara, United Republic of Tanzania

${ }^{5}$ Emergency \& Essential Surgical Care program, Department Essential Health Technologies, WHO, Geneva, Switzerland

${ }^{6}$ Ministry of Health and Social Welfare, United Republic of Tanzania
}

Acknowledgements The following members of GIEESC in Tanzania were instrumental in obtaining the reported data: Professor Neboth Mbembati, Professor Victor Mwafongo, Dr Paul Mareale, Dr Jasper Mbwambo, Dr Amani Malima, Dr Mtumwa Ibrahim and Dr Rashid Mayoka.

Contributors TP analysed and interpreted the data, drafted the article, revised it and finally approved the submitted version. $\mathrm{HC}$ contributed to conception and design, acquisition and interpretation of data, made critical revisions and finally approved the submitted version. PK contributed to acquisition of data, made critical revisions and finally approved the submitted version. AM contributed to acquisition of data, made critical revisions and finally approved the submitted version. GS contributed to acquisition of data, made critical revisions and finally approved the submitted version. LN contributed to conception and design, interpretation of data, made critical revisions and finally approved the submitted version. SG contributed to conception and design, interpretation of data, made critical revisions and finally approved the submitted version. DHM contributed to acquisition of data, made critical revisions and finally approved the submitted version. MC contributed to conception and design, acquisition and interpretation of data, helped draft the article, made critical revisions and finally approved the submitted version.

\section{Funding None.}

Disclaimer The authors include staff members of WHO. They are responsible for the views expressed in this publication and do not necessarily represent the decisions or stated policy of WHO.

Competing interests None.

Provenance and peer review Not commissioned; externally peer reviewed.

Data sharing statement No additional data are available, all has been included.

\section{REFERENCES}

1. Debas HT, Gosselin R, McCord C, et al. Surgery. In: Jamison D, ed Disease Control Priorities in Developing Countries. 2nd edn. New York: Oxford University Press, 2006:1245-59.

2. Weiser TG, Regenbogen SE, Thompson KD, et al. An estimation of the global volume of surgery: a modeling strategy based on available data. Lancet 2008;372:139-44.

3. World Health Organization (Internet site). Geneva: World Health Organization. http://www.who.int/countries/tza/en/ (accessed 18 Feb 2011).

4. Mwakyusa D. The National Road Map Strategic Plan to Accelerate Reduction of Maternal, Newborn and child deaths in Tanzania 2008-2015. Dar es Salaam, Tanzania: Annual Summit of MoHSW, 2008:1-13.

5. Emergency and essential surgical care (Internet site). Geneva: World Health Organization. http://www.who.int/surgery (accessed 18 Feb 2011).

6. Maternal mortality in 2005: estimates developed by WHO, UNICEF, UNFPA and the World Bank. Geneva: World Health Organization, 2007. http://www.who.int/reproductive-health/publications/ maternal mortality 2005/mme_2005.pdf

7. Cherian $\bar{M}$, Choo S, Wilson I, et al. Building and retaining the neglected anaesthesia health workforce: is it crucial for health systems strengthening through primary health care? Bull WHO 2010;88:637-9.

8. Laxminarayan R, Mills AJ, Breman JG, et al. Advancement of global health: key messages from the Disease Control Priorities Project. Lancet 2006;367:1193-208.

9. Gosselin R, Thind A, Bellardinelli A. Cost/DALY averted in a small hospital in Sierra Leone: what is the Relative Contribution of Different services? World J Surg 2006;30:505-11.

10. McCord C, Chowdhury J. A cost-effective small hospital in Bangladesh: what it can mean for emergency obstetric care. Int $J$ Gynaecol Obstet 2003;81:83-92.

11. Belle J, Cohen H, Shindo N, et al. Influenza preparedness in lowresource settings: a look at oxygen delivery in 12 African countries. $J$ Inf Dev Count 2010;4:419-24.

12. Hedges JP, Mock CN, Cherian MN. The Political Economy of emergency and essential surgery in global health. World $J$ Surg 2010;34:2003-6. 